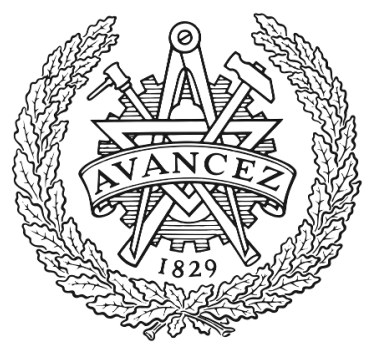

CHALMERS

UNIVERSITY OF TECHNOLOGY

\title{
Scaling laws of the Kondo problem at finite frequency
}

Downloaded from: https://research.chalmers.se, 2023-04-26 11:39 UTC

Citation for the original published paper (version of record):

Bruhat, L., Viennot, J., Dartiailh, M. et al (2018). Scaling laws of the Kondo problem at finite frequency. Physical Review B, 98(7). http://dx.doi.org/10.1103/PhysRevB.98.075121

N.B. When citing this work, cite the original published paper. 


\title{
Scaling laws of the Kondo problem at finite frequency
}

\author{
L. E. Bruhat, ${ }^{1,2, *}$ J. J. Viennot, ${ }^{1,3}$ M. C. Dartiailh, ${ }^{1}$ M. M. Desjardins, ${ }^{1}$ A. Cottet, ${ }^{1}$ and T. Kontos ${ }^{1, \dagger}$ \\ ${ }^{1}$ Laboratoire Pierre Aigrain, Ecole Normale Supérieure-PSL Research University, CNRS, Universités Pierre et Marie Curie-Sorbonne \\ Universités, Université Denis Diderot-Sorbonne Paris Cité, 24, rue Lhomond, 75231 Paris Cedex 05, France \\ ${ }^{2}$ Microtechnology and Nanoscience, Chalmers University of Technology, Kemivgen 9, SE-41296 Gothenburg, Sweden \\ ${ }^{3}$ JILA and Department of Physics, University of Colorado, Boulder, Colorado 80309, USA
}

(Received 9 December 2017; revised manuscript received 23 February 2018; published 9 August 2018)

\begin{abstract}
Driving a quantum system at finite frequency allows one to explore its dynamics. This has become a well-mastered resource for controlling the quantum state of two-level systems in the context of quantum information processing. However, this can also be of fundamental interest, especially with many-body systems which display an intricate finite-frequency behavior. In condensed matter, the Kondo effect epitomizes strong electronic correlations, but the study of its dynamics and the related scaling laws has remained elusive so far. Here, we fill this gap by studying a carbon-nanotube-based Kondo quantum dot at half filling driven by a microwave signal. Our findings not only confirm long-standing theoretical predictions but also allow us to establish a simple ansatz for the scaling laws on the Kondo problem at finite frequency. More generally, our technique opens a path for understanding the dynamics of complex quantum dot circuits in the context of quantum simulation of strongly correlated electron fluids.
\end{abstract}

DOI: 10.1103/PhysRevB.98.075121

\section{INTRODUCTION}

The use of quantum dot circuits to study the physics of interacting electrons in a controlled way is now well established. Such nanoscale circuits are ideally suited to study correlation effects, thanks to their tunability [1-5] and natural interface with high-frequency signals [6]. Correlation effects are deeply related to Kondo physics, which remains a major topic of condensed matter. While there have been a lot of experiments related to this phenomenon in the static regime, there are only few experiments in the dynamic regime, which can be accessed, for example, through the study of electronic transport under high-frequency driving.

The study of high-frequency-driven quantum dots is almost as old as the advent of quantum dot circuits, due to the interest in photon-assisted tunneling (PAT). Most of the works have focused on the Coulomb blockade regime [7,8], for which Coulomb conductance peaks appear when dot charge fluctuations are energetically allowed. In this case, PAT processes essentially produce sidebands around the Coulomb peaks, located at source-drain bias voltages $V_{\mathrm{SD}} \approx \pm h f / e$ where $f$ is the frequency of the microwave radiation and $e$ is the elementary charge. These experiments, which are well understood, have even been generalized to terahertz frequencies or to on-chip detection schemes for quantum noise [9-11].

In contrast, on the experimental side, PAT has raised little attention in strongly correlated quantum dot circuits. However, strong interactions lead to a rich dynamical phenomenology [12-20]. A radiation at frequency $f$ is a natural tool to

\footnotetext{
*Author to whom correspondence should be addressed: bruhat@chalmers.se

${ }^{\dagger}$ Author to whom correspondence should be addressed: kontos@lpa.ens.fr
}

study such finite-frequency processes. For instance, the Kondo dynamics, which depends on the Kondo temperature $T_{K}$, could be scrutinized by using $k_{B} T_{K} \sim h f$, in principle. However, even the simple question of whether or not sidebands appear around the Kondo resonance peak is intricate. Whereas there is a rich theoretical literature [12-20], only four experiments have been carried out on that topic in the last decade [21-24]. Noticeably, contradicting results were found on the existence [22,23] or not [21] of microwave-induced sidebands for a Kondo peak. In addition, the predictions of a scaling behavior [19], which is the hallmark of the Kondo effect [2,3,25], could not be tested experimentally [24]. Strikingly, although the dot microscopic description depends on many parameters, its reduced zero-voltage conductance is expected to depend only on the reduced amplitude $e V_{\mathrm{ac}} / k_{B} T_{K}$ and frequency $h f / k_{B} T_{K}$ of the oscillating voltage applied to the dot.

This lack of quantitative test is due to the experimental challenge of controlling both the excitation amplitude $V_{\mathrm{ac}}$ and the excitation frequency $f$ over a broad band. To cope with these difficulties, previous experimental studies of the ac Kondo effect either worked at a fixed frequency [22] or partly postulated the Kondo response itself to justify their microwave amplitude calibration [21,24]. These facts probably explain the lack of clear consensus on the underlying physics of photon-assisted tunneling of a Kondo dot.

The key achievement of our work is precisely to provide a quantitative amplitude-frequency characterization of the ac Kondo effect. We use a carbon-nanotube-based quantum dot at half filling exposed to a microwave radiation. We find that the existence of photon-assisted tunneling peaks for the ac Kondo problem depends on the excitation frequency used. In contrast to previous works, we use an independent calibration method for $V_{\mathrm{ac}}$, based on the adiabatic response of the current in the Coulomb blockade regime (see Appendix B). This allows us to 

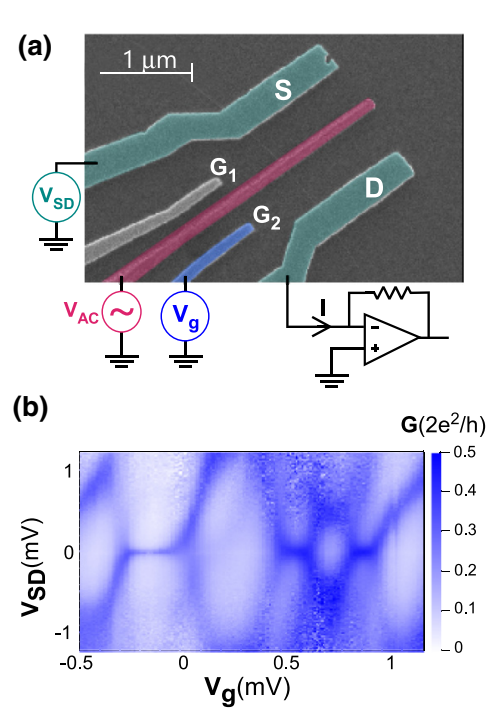

(c)

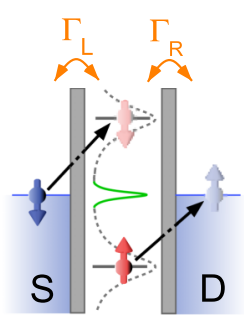

(d)

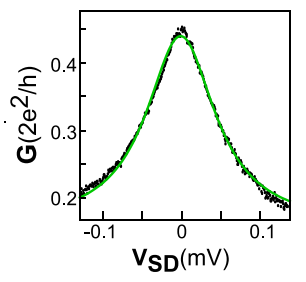

FIG. 1. (a) Scanning electron microscope picture of our device. It behaves as a single dot, which can be biased by applying a dc voltage $V_{\mathrm{SD}}$ on the source electrode, while the current $I$ is measured through the drain electrode. A dc gate voltage $V_{G}$ is applied on a side gate. A microwave excitation $V_{\text {ac }}$ at frequency $f$ can be applied on the central top gate. (b) Conductance color plot versus gate and bias voltage showing characteristic Kondo ridges. (c) Example of second-order tunnel process which flips the dot spin. The constructive interference of such processes at all orders gives rise to the Kondo peak for $V_{\mathrm{SD}}=0$. (d) Plot of Kondo resonance conductance peak at equilibrium $\left(V_{\mathrm{ac}}=0\right)$. The black dots are data and the solid green line is a Lorentzian fit giving $k_{B} T_{K}=32.7 \mu \mathrm{eV}, G_{\mathrm{OFF}}=0.281\left(2 e^{2} / h\right)$, and $G_{\mathrm{BG}}=0.159\left(2 e^{2} / h\right)$ [see Eq. (A1) in Appendix A].

study the full amplitude-frequency map of the Kondo peak at zero bias, and to make a quantitative test of the long-standing theoretical predictions of Kaminski, Nazarov, and Glazman [19]. In particular, we observe an apparently paradoxical prediction of the theory: in the case of bias excitation, the higher the frequency $f$, the lower the effect on the Kondo peak at a constant microwave amplitude $V_{\mathrm{ac}}$. We finally compare two Kondo peaks with different $T_{K}$ 's in order to perform a full test of the scaling properties of the Kondo effect at finite frequency. We are able to test simple analytical expressions for the scaling properties of the Kondo resonance using an ansatz adapted from the work of Kaminski, Nazarov, and Glazman.

\section{EXPERIMENTAL SETUP}

Our device is a carbon-nanotube quantum dot circuit embedded in a coplanar waveguide microwave cavity [Fig. 1(a)]. A bias voltage $V_{\mathrm{SD}}$ can be applied to one of the two Pd contact electrodes (emerald green). A dc gate voltage $V_{g}$ applied on a side gate electrode (blue) tunes the dot orbital energy. A central top gate electrode (pink) is used to apply a microwave signal to the device. Our calibration shows that although it is connected to the top gate electrode, it predominantly results in a source and drain ac bias voltage $V_{\text {ac }}$ (see Appendix B). Our setup measures the current $I$ and the de differential conductance $G=$ $d I / d V_{\mathrm{SD}}$ at $20 \mathrm{mK}$. Figure $1(\mathrm{~b})$ shows a $V_{\mathrm{SD}}-V_{g}$ conductance color plot in the absence of microwave excitation $\left(V_{\mathrm{ac}}=0\right)$. It displays zero-bias ridges characteristic of the Kondo regime. (a)

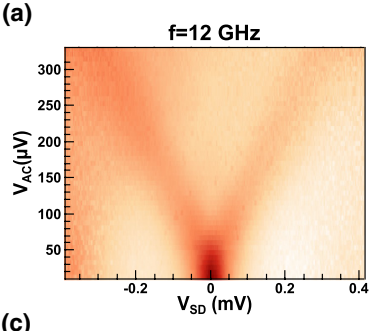

(b)

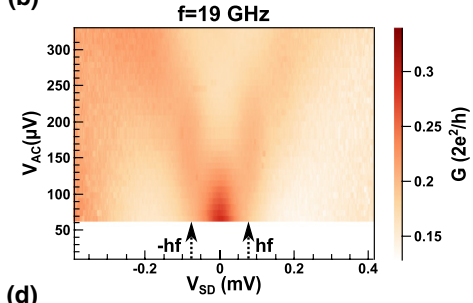

(c)

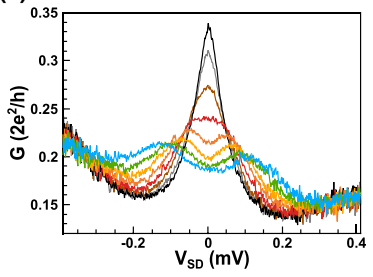

(d)

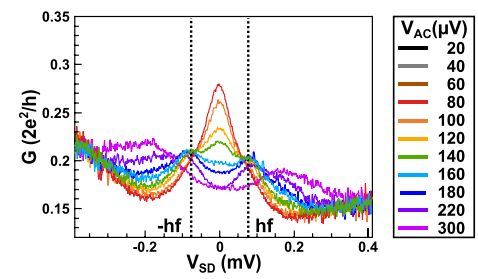

FIG. 2. Color plot of the conductance versus bias voltage $V_{\mathrm{SD}}$ and excitation voltage $V_{\text {ac }}$ with excitation frequency (a) $f=12 \mathrm{GHz}$ and (b) $f=19 \mathrm{GHz}$, corresponding to reduced frequencies (a) $h f / k_{B} T_{K}=1.8$ and (b) $h f / k_{B} T_{K}=2.8 . k_{B} T_{K}, G_{\mathrm{OFF}}$, and $G_{\mathrm{BG}}$ for this data set differ slightly from the data shown in Fig. 1(b) and their values are given in Fig. 8 (file number 18). Linear cuts of the data shown in (c) panel (a) and (d) panel (b). The lines' color encodes the excitation voltage, and the color code applies to both frequencies.

One of the Kondo resonance conductance peaks [Fig. 1(d)] corresponds to a Kondo temperature $T_{K} \sim 380 \mathrm{mK}$ extracted from a Lorentzian fit (see Appendix A). Note that typical Kondo temperatures in carbon nanotubes rather lie in the 1-10 $\mathrm{K}$ range, but a lower $T_{K}$ is advantageous to explore the Kondo dynamics in the relevant regime $k_{B} T_{K} \sim h f$ with accessible gigahertz frequencies.

\section{KONDO PEAK UNDER MICROWAVE IRRADIATION}

We now apply a finite microwave excitation $V_{\text {ac }}$ and study how the Kondo resonance conductance peak is modified, for two excitation frequencies, $f=12 \mathrm{GHz}$ and $f=19 \mathrm{GHz}$, for which the microwave excitation is dominantly coupled to the source-drain bias of the dot (see Appendix A). Our measurements confirm the phenomenology observed in previous experiments (Fig. 2). The stronger the microwave excitation, the lower the Kondo resonance peak [Figs. 2(c) and 2(d)]. Above a certain excitation voltage, several peaks are visible. For $f=12 \mathrm{GHz}$, we observe a linear splitting of the Kondo resonance versus $V_{\text {ac }}$ [Figs. 2(a) and 2(c)], which corresponds to the adiabatic regime. In contrast, for $f=19$ $\mathrm{GHz}$, three peaks can be resolved simultaneously [green curve on Fig. 2(d)], and the two side peaks remain locked at $\pm h f$ over a certain range of excitation voltage [Figs. 2(b) and 2(d)]. These satellites are the manifestation of photoinduced Kondo resonances. Figures 2(c) and 2(d) also show that the conductances at the Kondo resonance $\left(V_{\mathrm{SD}}=0\right)$ with and without applied microwave excitation $\left(G_{\mathrm{ON}}\right.$ and $\left.G_{\mathrm{OFF}}\right)$ clearly differ and that the variation of $G_{\mathrm{ON}}$ with $V_{\mathrm{ac}}$ quantitatively differs for $f=12 \mathrm{GHz}$ and $f=19 \mathrm{GHz}$.

Using our calibration, we obtain the experimental map of the conductance ratio $G_{\mathrm{ON}} / G_{\mathrm{OFF}}$ with and without microwave excitation as a function of the scaled amplitude $e V_{\mathrm{ac}} / k_{B} T_{K}$ 
(a)

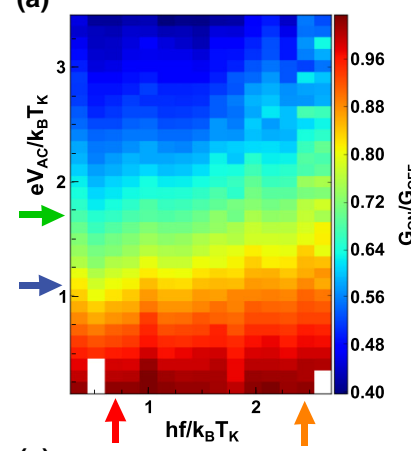

(c)

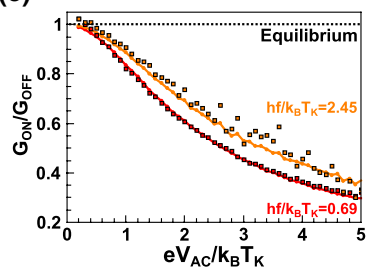

(b)

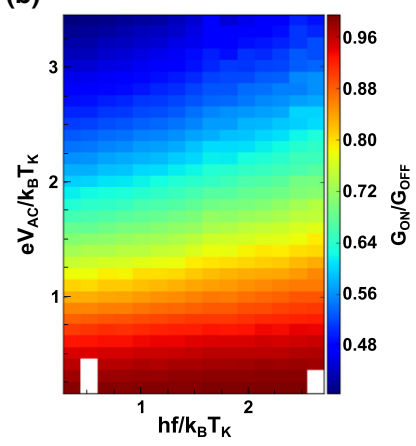

(d)

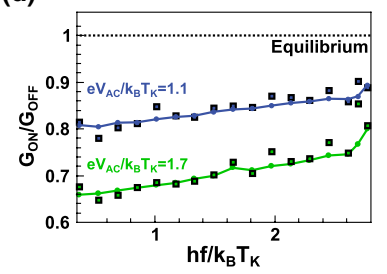

FIG. 3. (a) Experimental averaged normalized conductance color plot $G_{\mathrm{ON}} / G_{\mathrm{OFF}}$ as a function of excitation scaled amplitude $e V_{\text {ac }} / k_{B} T_{K}$ and scaled frequency $h f / k_{B} T_{K}$. (b) Calculated map of $G_{\mathrm{ON}} / G_{\mathrm{OFF}}$ using the ansatz formula (1). (c) Linear cuts at $h f / k_{B} T_{K}=0.69$ (red) and $h f / k_{B} T_{K}=2.45$ (orange). (d) Linear cuts at $e V_{\mathrm{ac}} / k_{B} T_{K}=1.1$ (blue) and $e V_{\mathrm{ad}} / k_{B} T_{K}=1.7$ (green). In (c) and (d), squares correspond to data points, while dots linked by solid lines correspond to the ansatz. The dashed black line indicates the equilibrium reference $\left(V_{\mathrm{ac}}=0\right)$.

and scaled frequency $h f / k_{B} T_{K}$ [Fig. 3(a); see Appendix A]. While $G_{\mathrm{ON}} / G_{\mathrm{OFF}}$ decreases with $V_{\mathrm{ac}}$, it increases with $f$. These trends appear even more clearly on the map line cuts plotted in Figs. 3(c) and 3(d). The higher the amplitude, the further the conductance at the Kondo resonance gets away from the equilibrium value, up to data noise. In contrast, the higher the frequency, the closer the Kondo conductance gets to its equilibrium value. Both behaviors were predicted by Kaminski, Nazarov, and Glazman for an ac excitation of the bias voltage, in the limits $f \rightarrow 0$ and $V_{\mathrm{ac}} \rightarrow 0$, respectively. From their theoretical work, we build the following ansatz to quantitatively describe our data for finite values of $f$ and $V_{\text {ac }}$ :

$$
\begin{aligned}
& \frac{G_{\mathrm{ON}}}{G_{\mathrm{OFF}}}\left(\frac{e V_{\mathrm{ac}}}{k_{B} T_{K}}, \frac{h f}{k_{B} T_{K}}, G_{\mathrm{OFF}}\right) \\
& =\frac{1}{\sqrt{1+\frac{3}{8}\left(\frac{e V_{\mathrm{ac}}}{k_{B} T_{K}}\right)^{2} \alpha\left(\frac{h f}{k_{B} T_{K}}, G_{\mathrm{OFF}}\right)}}
\end{aligned}
$$

with

$$
\alpha\left(\frac{h f}{k_{B} T_{K}}, G_{\mathrm{OFF}}\right)=\frac{1}{\sqrt{1+\left(\frac{16}{3} \frac{a}{\pi} \frac{G_{\mathrm{OFF}}}{2 e^{2} / h} \frac{k_{B} T_{K}}{h f}\right)^{-2}}},
$$

where $a \sim 1$ is a dimensionless parameter. More precisely, this expression bridges the analytical expressions of $G_{\mathrm{ON}} / G_{\mathrm{OFF}}$ in the adiabatic limit [Eq. (76) of Ref. [19]] and in the low-amplitude high-frequency limit [Eq. (74) of Ref. [19]].

(a)

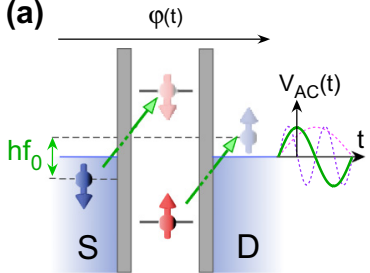

(b)
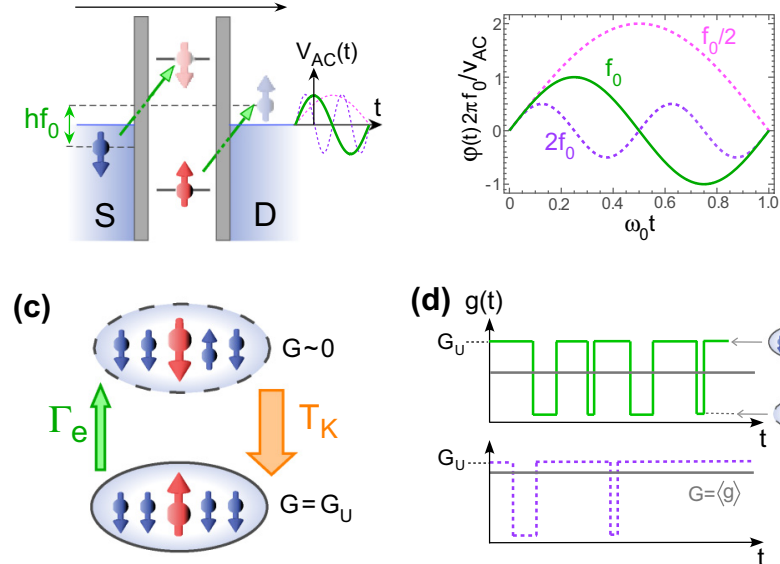

(d) $g(t)$

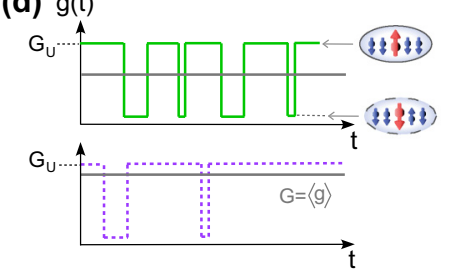

FIG. 4. (a) Sketch of a quantum dot with an ac bias voltage $V_{\mathrm{ac}}(t)=V_{\mathrm{ac}} \cos (2 \pi f t)$. Due to this modulation, photons can trigger second-order tunnel processes, represented here by the pair of green arrows, which flip the dot spin and leave a high-energy electron in the reservoirs. The three colored solid and dotted lines represent $V_{\mathrm{ac}}(t)$ for different values of excitation frequency $f$ with the same $V_{\text {ac }}$. This color code applies to all panels. (b) Plot of the phase difference $\varphi(t)$ between the electronic states in the source and drain, as a function of time. (c) Representation of the circuit dynamics. The dot leaves the Kondo ground state with a rate $\Gamma_{e}$ and relaxes to the Kondo ground state again with a rate $k_{B} T_{K} / \hbar$. (d) Time dependence of the dot conductance $g(t)$, which reaches $G_{U}$ in the Kondo state and is much smaller otherwise. The average conductance $G=\langle g(t)\rangle$ measured in the experiment is closer to $G_{U}$ for large values of $f$ for which $\Gamma_{e}$ is smaller.

Strikingly, our ansatz is able to reproduce the full amplitudefrequency map of $G_{\mathrm{ON}} / G_{\mathrm{OFF}}$ with a single adjustable parameter $a=4.4$ [Fig. 3(b)]. A $10 \%$ global correction has been applied to amplitudes $\left(\tilde{V}_{\mathrm{ac}}=1.1 V_{\mathrm{ac}}\right)$ to account for absolute calibration uncertainty. The good agreement between our ansatz and our measurements can be better appreciated from the linear cuts displayed in Figs. 3(c) and 3(d).

Can we find a physical picture for the counterintuitive frequency dependence we observe? Indeed one would rather think that higher frequencies would represent more energetic perturbations for the Kondo cloud, thus resulting in more decoherence. However, our ac excitation affects the phase difference $\varphi$ between the electronic states in the source and drain electrodes, which becomes [26] $\varphi(t)=$ $e / \hbar \int^{t} V_{\mathrm{ac}} \cos \left(2 \pi f t^{\prime}\right) d t^{\prime}=e V_{\mathrm{ac}} \sin (2 \pi f t) / h f$ for $V_{\mathrm{dc}}=0$. These phase oscillations trigger inelastic co-tunneling processes in which the dot spin is flipped and an electron is sent above the Fermi level of the reservoirs, so that the circuit gets out of the highly conducting many-body singlet Kondo ground state [see Figs. 4(a) and 4(d)]. The rate $\Gamma_{e}$ associated to this decoherence process depends on the amplitude of the phase oscillations, which vanish at high excitation frequencies due to the $1 / f$ factor in the above equation [see Fig. 4(b)]. This simple fact explains that the Kondo cloud is less affected by higher frequencies. More quantitatively, at first order in $e V_{\mathrm{ac}} / h f$, the two spin states of the dot are coupled by an oscillating matrix element with amplitude $J_{0} e V_{\mathrm{ac}} / h f$ [see Eq. (18) of Ref. [19]], with $J_{0}$ the spin-exchange factor of the 


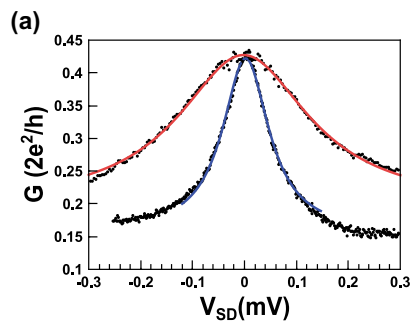

(b)
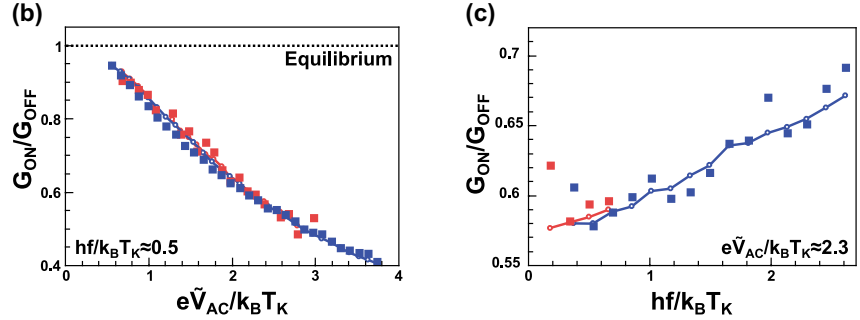

FIG. 5. (a) Plots of conductance versus bias voltage for narrow and broad Kondo resonances at equilibrium $\left(V_{\mathrm{ac}}=0\right)$. The black dots are data and the solid lines are Lorentzian fits yielding $k_{B} T_{K}=$ $33.3 \mu \mathrm{eV}$ for the narrow Kondo peak (blue) and $k_{B} T_{K}=95 \mu \mathrm{eV}$ for the broad Kondo peak (pink). Comparison of (b) scaled amplitude dependence and (c) scaled frequency dependence of $G_{\mathrm{ON}} / G_{\mathrm{OFF}}$ for broad (pink) and narrow (blue) Kondo peaks to investigate universality. Squares correspond to experimental data, while dots linked by solid lines correspond to the ansatz.

Kondo Hamiltonian in equilibrium. In the limit $\hbar \Gamma_{e} \ll k_{B} T_{K}$, which we consider for simplicity, decoherence is dominated by single-photon absorption processes such as the one represented in Fig. 4(a), and it involves initial reservoir states which must lie in a band of width $f$ below the Fermi energy of the leads, as visible from Fig. 4(a). Hence, from Fermi's golden rule, one gets $\Gamma_{e}=\left(J_{0} v e V_{\mathrm{ac}}\right)^{2} / \hbar^{2} f$ with $v$ the density of states in the leads. Once the dot spin is flipped, its conductance is negligible in comparison with the unitary conductance $G_{U}$ of the Kondo limit. Since the circuit relaxes to the Kondo ground state with a rate $k_{B} T_{K} / \hbar$, the average conductance reads $G=G_{U} /\left(1+\hbar \Gamma_{e} / k_{B} T_{K}\right) \simeq\left(1-\alpha V_{\mathrm{ac}}^{2} / f T_{K}\right) G_{U}$, with $\alpha=$ $2 \pi J_{0}^{2} e^{2} v^{2} / h k_{B}$, which corresponds to Eq. (74) of Ref. [19]. This reasoning explains the increase of $G$ with $f$ but also agrees with the decrease of $G$ with $V_{\text {ac }}$.

\section{SCALING LAWS}

Finally, we investigate the crucial issue of the ac Kondo predicted universality properties. Is $G_{\mathrm{ON}} / G_{\mathrm{OFF}}$ really governed by the reduced parameters $e V_{\mathrm{ac}} / k_{B} T_{K}$ and $h f / k_{B} T_{K}$ ? To address this question, we compare the ac response of Kondo resonances with significantly different Kondo temperatures. The peak fitted by the blue line in Fig. 5(a) is the "narrow" Kondo resonance, where the frequency-amplitude characterization of the previous section has been measured. The peak fitted by the pink line is three times broader and corresponds to $T_{K} \sim 1.1 \mathrm{~K}$. On the one hand, this factor of 3 is advantageous, as scaling should be tested unambiguously. On the other hand, it also means that the investigated scaled frequency range is correspondingly reduced. Indeed our microwave source delivers up to $20-\mathrm{GHz}$ frequencies, so we are limited to $h f / k_{B} T_{K} \lesssim$ 0.84. Following the color code of Fig. 5(a), Figs. 5(b) and
5 (c) show on the same graph $G_{\mathrm{ON}} / G_{\mathrm{OFF}}$ for the two peaks as a function of scaled amplitude and scaled frequency, respectively, at fixed scaled frequency $h f / k_{B} T_{K} \approx 0.5$ and scaled amplitude $e \tilde{V}_{\mathrm{ac}} / k_{B} T_{K} \approx 2.3$, respectively. By definition, the pink and blue ansatz curves merge, up to small discrepancies due to noise in $G_{\mathrm{OFF}}$ values. In Fig. 5(b), experimental data for both narrow and broad Kondo peaks collapse well onto the ansatz curve. This demonstrates the universal dependence of $G_{\mathrm{ON}} / G_{\mathrm{OFF}}$ on $V_{\mathrm{ac}}$ at low frequency. In Fig. 5(c), experimental data for the broad peak come down to four points, as explained above. They fall reasonably well on the narrow-peak data and confirm a flat adiabatic behavior at low scaled frequency. This is consistent with a universal frequency dependence, although universality in the pronounced frequency-dependant regime $h f / k_{B} T_{K}>1$ could not be investigated.

\section{CONCLUSION}

Our experiment realized on a carbon-nanotube (CNT)based quantum dot brings conclusions to several open issues of the ac Kondo problem. Using an independent in situ amplitude calibration, we provide a quantitative measurement of the amplitude-frequency dependence of the Kondo conductance with an ac bias. We observe or do not observe Kondo side peaks, depending on the excitation frequency used. The Kondo resonance is found to be less affected at higher excitation frequencies, in agreement with Kaminski et al. [19]. We describe our data quantitatively using an ansatz, which bridges expressions given in this paper for two limiting regimes of parameters. Measurement on two Kondo resonances with very different $T_{K}$ allows us to make tests of the universality of the Kondo behavior. We find a good scaling of $G_{\mathrm{ON}} / G_{\mathrm{OFF}}$ with the scaled amplitude $e V_{\mathrm{ac}} / k_{B} T_{K}$. In the more limited parameter range we could explore, our data are also consistent with a scaling of $G_{\mathrm{ON}} / G_{\mathrm{OFF}}$ with the scaled frequency $h f / k_{B} T_{K}$.

\section{ACKNOWLEDGMENTS}

We thank Leonid Glazman for fruitful discussions. We gratefully acknowledge J. Palomo, M. Rosticher, and A. Denis for technical support. The device has been made within the consortium Salle Blanche Paris Centre. This work is supported by ERC Starting Grant CirQys.

\section{APPENDIX A: EXPERIMENTAL DETAILS}

\section{Sample fabrication and measurement}

CNTs were grown on a separate quartz substrate containing mesas and transferred onto a high-resistivity $\mathrm{Si} / \mathrm{SiO}_{2}$ substrate using a method described in Ref. [27]. After localizing CNTs with a scanning electron microscope, the device electrodes were patterned using electronic nanolithography. The two outer normal electrodes (70 $\mathrm{nm}$ Pd) are connected to the CNT with a room-temperature resistance of $71 \mathrm{k} \Omega$. In contrast, the central superconducting electrode $(4 \mathrm{~nm} \mathrm{Pd} / 100 \mathrm{~nm} \mathrm{Al})$ is disconnected and acts as a top gate, on which we apply a microwave voltage to perform our study of the ac Kondo. Transport measurements as a function of the two side gates show parallel lines with a single slope, corresponding to a single dot behavior. Therefore, a single side gate is operated with 
gate voltage $V_{g}$. In addition to the dc current, the differential conductance $G$ is measured by synchronous detection using a small ac bias voltage of $10 \mu \mathrm{V}$ at $77.77 \mathrm{~Hz}$. The device is embedded in a $\mathrm{Nb}$ coplanar waveguide resonator, which was not used in this work as transmission measurements showed no coupling between the two systems.

\section{Fitting the Kondo resonance at equilibrium}

We fit the Kondo resonance conductance peak in absence of microwave excitation by an offset Lorentzian curve [Figs. 1(d) and 5(a)]:

$$
G=G_{\mathrm{BG}}+\frac{G_{\mathrm{OFF}}}{1+\frac{3}{8}\left(\frac{e V_{\mathrm{SD}}-x_{c}}{k_{B} T_{K}}\right)^{2}} .
$$

The Lorentzian peak is centered on $x_{c}$ with an amplitude $G_{\text {OFF }}$ and a width setting our practical definition of $T_{K}$. The parametrization is chosen to recover the $\mathrm{SU}(2)$ coefficient of the (quadratic) Fermi liquid description at low energy [28]. The presence of an offset background conductance $G_{\mathrm{BG}}$ is commonly observed in the Kondo regime [29]. For instance, this could be a contribution of co-tunneling, which would evolve over the scale $U \approx 1 \mathrm{meV} \gg k_{B} T_{K}$, which can be considered flat over the Kondo resonance versus bias voltage.

\section{Microwave amplitude calibration}

Our in situ calibration method consists in measuring the current rectification under microwave excitation in the Coulomb regime. Considering our parameter range $(U \approx 1 \mathrm{meV} \gg h f$ and $\Gamma \approx 200 \mu \mathrm{eV}>2.5 h f$ ), we can reasonably assume that the ac excitation simply modulates the dc current-voltage characteristic $I(V)$. This leads to the following expression of the measured current:

$$
\underbrace{\left\langle I\left(V_{\mathrm{SD}}+V_{\mathrm{ac}} \cos \omega t\right)\right\rangle}_{I_{\mathrm{ON}}}=\underbrace{I\left(V_{\mathrm{SD}}\right)}_{I_{\mathrm{OFF}}}+\frac{1}{4} \frac{d^{2} I}{d V_{\mathrm{SD}}^{2}} V_{\mathrm{ac}}^{2},
$$

which is expressed to lowest order in the excitation voltage on the sample $V_{\mathrm{ac}}$. This is valid if $V_{\mathrm{ac}}$ explores the linear part of the characteristic $G\left(V_{\mathrm{SD}}\right)$, leading to $V_{\mathrm{ac}} \ll U \approx 1 \mathrm{meV}$. For each frequency a proportionality coefficient $c(f)$ links the sample voltage to the source voltage: $V_{\mathrm{ac}}=c(f) V_{\mathrm{ac}, \text { source }}$. The $c(f)$ values and the detailed procedure to obtain them are given in Appendix B (Fig. 6). Note that Eq. (A2) holds only if the microwave excitation mainly acts as an ac bias voltage. This is the case of most excitation frequencies, where the calibration coefficient $c(f)$ is independent of the gate voltage. Frequencies with a gate-dependent $c(f)$ correspond to a mixed ac bias-ac gate excitation regime, which is not the well-controlled situation we want to study. These frequencies are excluded from the frequency set used for the ac Kondo characterization (for more details, see Appendix C and Fig. 7).

\section{Scaled frequency-amplitude conductance map building procedure}

The experimental scaled frequency-amplitude conductance map shown in Fig. 3(a) (resp. in Fig. 9) is built from a database (a)

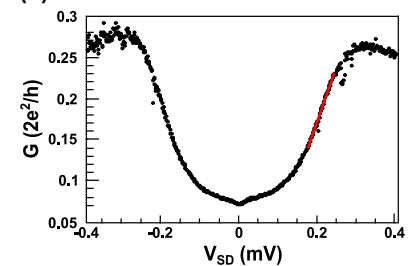

(c)

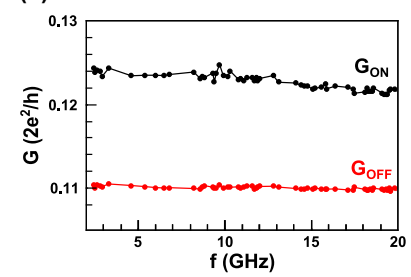

(b)

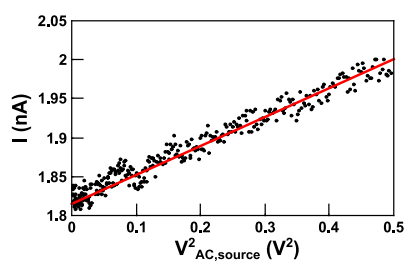

(d)

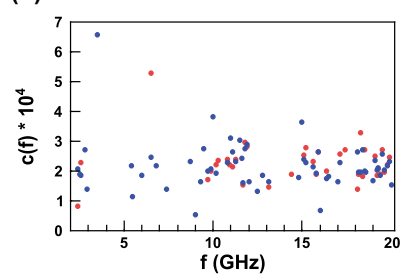

FIG. 6. (a) The local slope of the conductance $G$ versus bias voltage gives $\frac{d^{2} I}{d V_{\mathrm{SD}}^{2}}$. Here around $V_{\mathrm{SD}}=0.207 \mathrm{mV}, \frac{d^{2} I}{d V_{\mathrm{SD}}^{2}}=1.0 \times 10^{-1}$ $\mathrm{A} / \mathrm{V}^{2}$. (b) Knowing this value, the slope $p$ of the current $I$ as a function of the square source amplitude $V_{\text {ac,source }}$ in the linear regime yields the amplitude calibration coefficient $c(f)=\sqrt{4 p / \frac{d^{2} I}{d V_{\mathrm{SD}}^{2}}}$. Here for $f=$ $20 \mathrm{GHz} p=0.372 \mathrm{nA} / \mathrm{V}^{2}$; hence $c(f=20 \mathrm{GHz})=1.2 \times 10^{-4}$. (c) An iterative relative amplitude calibration. For each frequency the conductance is successively measured with and without microwave excitation. Calibration coefficients $c_{n}(f)$ are used to set a $V_{\text {ac }} \approx$ $200 \mu \mathrm{V}$ for each frequency. The new coefficient set $c_{n+1}(f)$ is deduced from Eq. (B3) with $G$ instead of $I$. (d) The calibration coefficients $c(f)$ used to calculate the excitation amplitudes $V_{\text {ac }}$ applied to the narrow (blue dots) and broad (pink dots) Kondo peaks.

of 5189 sets (resp. 189) of ( $\left.f, V_{\mathrm{ac}, \text { source }}, G\right)$ gathering all measurements of the microwave response of the "narrow" blue (resp. "broad" pink) Kondo peak in Fig. 5(a). The various measurement types and data extraction procedures are detailed in the Appendixes. The amplitude calibration coefficients $c(f)$ are used to calculate the excitation amplitude $V_{\mathrm{ac}}$ from $V_{\mathrm{ac} \text {,source. }} G_{\mathrm{BG}}, G_{\mathrm{OFF}}$, and $T_{K}$ are obtained from the equilibrium Kondo resonance fit [see Eq. (A1) above] before each data acquisition (see Fig. 8 for parameters). This allows us to calculate the excitation scaled frequency $h f / k_{B} T_{K}$ and scaled amplitude $e V_{\mathrm{ac}} / k_{B} T_{K}$. The conductance under excitation $G_{\mathrm{ON}}$ is obtained by subtracting the background

(a)

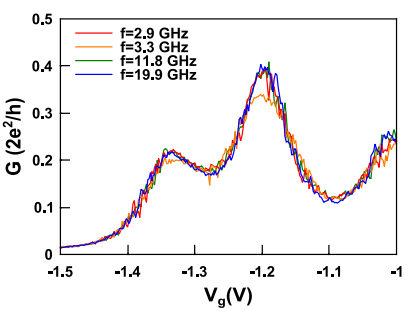

(b)

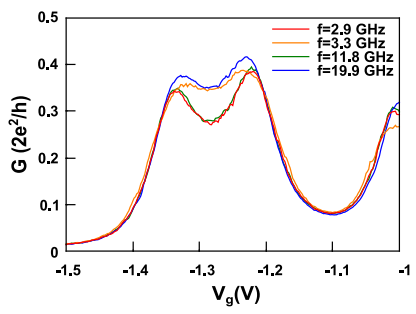

FIG. 7. (a) Conductance measurement at finite bias $\left(V_{\mathrm{SD}}=\right.$ $203 \mu \mathrm{V})$ and under ac excitation $V_{\mathrm{ac}}=100 \mu \mathrm{V}$. Curves at $f=2.9$, 11.8 , and $19.9 \mathrm{GHz}$ superpose at all gate voltages, while the $f=3.3$ $\mathrm{GHz}$ curve does not. (b) Conductance measurement at zero bias under ac excitation $\left(V_{\mathrm{ac}}=100 \mu \mathrm{V}\right)$ for the same frequencies. 


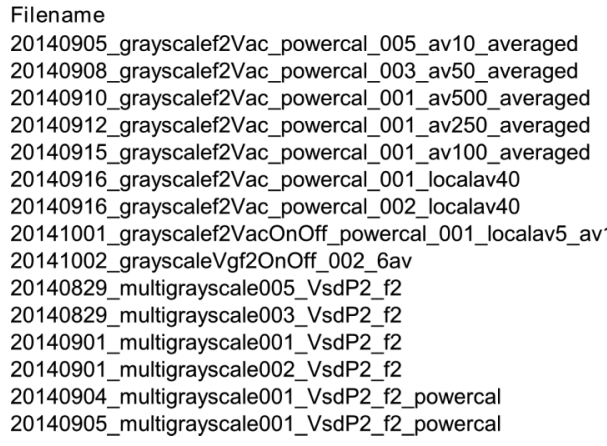

$\begin{array}{ccccccccc}\# & \mathrm{~T}_{\mathrm{K}}(\mu \mathrm{eV}) & \mathrm{G}_{\mathrm{BG}}\left(2 \mathrm{e}^{2} / \mathrm{h}\right) & \mathrm{G}_{\mathrm{OFF}}\left(2 \mathrm{e}^{2} / \mathrm{h}\right) & \mathrm{x}_{\mathrm{c}}(\mathrm{mV}) & \text { std-Tk } & \text { std-GBG } & \text { std-GOff } & \text { std-xc } \\ 0 & 24.6 & 0.138 & 0.2 & 0.127 & 0 & 0 & 0 & 0 \\ 1 & 29.7 & 0.16 & 0.266 & 0.132 & 0 & 0 & 0 & 0 \\ 2 & 30.8 & 0.164 & 0.256 & 0.128 & 0 & 0 & 0 & 0 \\ 3 & 33.3 & 0.156 & 0.266 & 0.122 & 0 & 0 & 0 & 0 \\ 4 & 33.1 & 0.156 & 0.274 & 0.127 & 0 & 0 & 0 & 0 \\ 5 & 32.7 & 0.159 & 0.281 & 0.128 & 0 & 0 & 0 & 0 \\ 6 & 32.7 & 0.159 & 0.281 & 0.128 & 0 & 0 & 0 & 0 \\ 9 & 95 & 0.196 & 0.229 & 0.118 & 0 & 0 & 0 & 0 \\ 12 & 95 & & 0.229 & 0.118 & 0 & 0 & 0 & 0 \\ 13 & 28.9 & 0.136 & 0.193 & 0.124 & 0.6 & 0.003 & 0.001 & 0.003 \\ 14 & 21.8 & 0.169 & 0.265 & 0.124 & 0.9 & 0.01 & 0.005 & 0.001 \\ 15 & 29 & 0.141 & 0.194 & 0.126 & 0.3 & 0.003 & 0.004 & 0.004 \\ 16 & 29.6 & 0.145 & 0.193 & 0.123 & & & & \\ 18 & 28.2 & 0.134 & 0.195 & 0.123 & 0.4 & 0.0005 & 0.002 & 0.0006 \\ 19 & 29.9 & 0.138 & 0.215 & 0.12 & 2 & 0.01 & 0.03 & 0.002\end{array}$

FIG. 8. Table of Kondo equilibrium parameters associated with each data file.

conductance $G_{\mathrm{BG}}$. We assume $G_{\mathrm{BG}}$ is not changed by the excitation, as $\left(h f, e V_{\mathrm{ac}}\right) \ll U$. This assumption is validated by Figs. 2(c) and 2(d), where conductance curves for all $V_{\mathrm{ac}}$ values merge at high bias voltage. For comparison between different data sets and/or between data and theory, it is relevant to normalize $G_{\mathrm{ON}}$ by the equilibrium conductance $G_{\mathrm{OFF}}$ : $G_{\mathrm{ON}} / G_{\mathrm{OFF}}=\left(G-G_{\mathrm{BG}}\right) / G_{\mathrm{OFF}}$. Finally, because data points are not uniformly spaced in the $\left(e V_{\mathrm{ac}} / k_{B} T_{K}\right)-\left(h f / k_{B} T_{K}\right)$ plane, we choose a regular grid and obtain the map matrix by averaging data points in the same cell. Cells with no data points remain blank. Here the cell size is 0.16 in $h f / k_{B} T_{K}$ and 0.1 in $e V_{\text {ac }} / k_{B} T_{K}$. The ansatz scaled frequency-amplitude conductance maps are built following the same procedure except that the values for $G_{\mathrm{ON}} / G_{\mathrm{OFF}}$ are calculated from the ansatz formula (1) given in the main text, using experimental values for $e V_{\mathrm{ac}} / k_{B} T_{K}, h f / k_{B} T_{K}$, and $G_{\mathrm{OFF}}$. This explains why the ansatz curves are not perfectly smooth.

\section{APPENDIX B: IN SITU MICROWAVE AMPLITUDE CALIBRATION}

Our in situ calibration method consists in measuring the current rectification under microwave excitation in the Coulomb regime. Considering our parameter range $(U \approx 1 \mathrm{meV} \gg h f$ and $\Gamma \approx 200 \mu \mathrm{eV}>2.5 \mathrm{hf}$ ), we can reasonably assume that the ac excitation simply modulates the dc current-voltage characteristic $I(V)$. This leads to the following expression of the measured current:

$$
\underbrace{\left\langle I\left(V_{\mathrm{SD}}+V_{\mathrm{ac}} \cos \omega t\right)\right\rangle}_{I_{\mathrm{ON}}}=\underbrace{I\left(V_{\mathrm{SD}}\right)}_{I_{\mathrm{OFF}}}+\frac{1}{4} \frac{d^{2} I}{d V_{\mathrm{SD}}^{2}} V_{\mathrm{ac}}^{2},
$$

which is expressed to lowest order in the excitation voltage on the sample, $V_{\text {ac }}$. This is valid if $V_{\text {ac }}$ explores the linear part of the characteristic $G\left(V_{\mathrm{SD}}\right)$, leading to $V_{\mathrm{ac}} \ll U \approx 1 \mathrm{meV}$. For each frequency a proportionality coefficient $c(f)$ links the sample voltage to the source voltage: $V_{\mathrm{ac}}=c(f) V_{\mathrm{ac} \text {, source }}$.

Two calibration methods were combined to obtain the values of $c(f)$. The first method provides a reliable absolute calibration. In the absence of excitation $\left(V_{\mathrm{ac}}=0\right), \frac{d^{2} I}{d V_{\mathrm{SD}}^{2}}$ is measured at $V_{\mathrm{SD}, 0}$ on the side of a Coulomb diamond. This is the local slope of the conductance as a function of bias voltage near $V_{\mathrm{SD}, 0}$, as depicted in Fig. 6(a). Figure 6(b) shows the current at $V_{\mathrm{SD}, 0}$ as a function of $V_{\mathrm{ac}}^{2}$. We focus on the low excitation regime, where the dependence is linear in agreement with Eq. (A2). Knowing $\frac{d^{2} I}{d V_{\mathrm{SD}}^{2}}$ at $V_{\mathrm{SD}, 0}$, the slope gives access to $c(f)$. Applying this method for each frequency is time consuming and the sample working point may change between first and last measured frequencies. Relative calibration is performed more rapidly and reliably using a second method. The source voltage is fixed and the frequency is swept. Current is successively measured with (on current $I_{\mathrm{ON}}$ ) and without (off current $I_{\mathrm{OFF}}$ ) microwave excitation for each frequency point. For frequencies $f_{1}$ and $f_{2}$ with $c(f)$ such that the lowest-order expansion (A2) is valid, calibration coefficients $c\left(f_{1}\right)$ and $c\left(f_{2}\right)$ are related by current rectification ratios:

$$
\frac{c\left(f_{2}\right)^{2}}{c\left(f_{1}\right)^{2}}=\frac{I_{\mathrm{ON}}\left(f_{2}\right)-I_{\mathrm{OFF}}}{I_{\mathrm{ON}}\left(f_{1}\right)-I_{\mathrm{OFF}}}
$$

Relative calibration can be refined by iterating the procedure. We apply a source voltage $V_{\text {ac, source }}(f)=V_{\mathrm{ac}, 0} / c_{n}(f)$ calculated to apply a constant excitation voltage $V_{\mathrm{ac}, 0} \approx 200 \mu \mathrm{V}$. The new relative calibration is deduced from

$$
\frac{c_{n+1}\left(f_{2}\right)^{2}}{c_{n+1}\left(f_{1}\right)^{2}}=\frac{c_{n}\left(f_{2}\right)^{2}}{c_{n}\left(f_{1}\right)^{2}} \frac{I_{\mathrm{ON}}\left(f_{2}\right)-I_{\mathrm{OFF}}}{I_{\mathrm{ON}}\left(f_{1}\right)-I_{\mathrm{OFF}}} .
$$

This relation holds replacing the current $I$ by the differential conductance $G$. Figure 6(c) shows the conductance on and off measurement, which defined the final relative calibration for some frequencies.

Figure 6(d) shows the coefficients $c(f)$ used to calibrate the amplitude $V_{\text {ac }}$ applied to the device for the study of the ac response of the narrow (blue dots) and broad (pink dots) Kondo peaks. Amplitude calibration for the narrow peak frequencies was done by combining absolute calibration and relative calibration methods described above. The resulting $c(f)$ are on the same order of magnitude. This is because they have been preselected to be; otherwise relative powers may vary over tens of decibels. We make this preselection to get a similar power range for all frequencies from the 43-dB dynamic power range of our microwave source. For the broad Kondo peak, calibration was performed by iterating a relative calibration obtained on the narrow peak. Calibration coefficients differ between broad and narrow peaks because a thermal cycling occurred between the two measurements runs. The thermal cycling did not change the calibration coefficients' order of magnitude, but relative calibration was modified, possibly because wire bonds moved. Note that Fig. 6(d) plots 
calibration coefficients only for frequencies kept after the frequency selection procedure, which we detail now.

\section{APPENDIX C: FREQUENCY SELECTION PROCEDURE}

Once calibration is done, we study its gate dependence. We measure the conductance as a function of gate voltage successively with excitation on $\left(V_{\mathrm{AC}}=100 \mu \mathrm{V}\right)$ and off. This is done at finite bias $V_{\mathrm{SD}}=203 \mu \mathrm{V}$ for all calibrated frequencies. As expected the curves coincide at $V_{g}=-1.095 \mathrm{~V}$, where calibration was performed. We keep only frequencies, which curves superpose entirely. For example we observe in Fig. 7(a) that frequencies 2.9, 11.8, and $19.9 \mathrm{GHz}$ match well at all gate voltages, while $3.3 \mathrm{GHz}$ does not. As a result $3.3 \mathrm{GHz}$ is excluded from the study of ac Kondo. Frequencies remaining after this procedure correspond to an ac excitation mainly applied on the dot bias voltage. When current rectification is gate dependent, the ac excitation very likely also applies to the dot energy level. Kaminski et al. [19] predicted an opposite frequency dependence of the ac Kondo conductance with this kind of coupling, compared to coupling to bias. $G_{\mathrm{ON}} / G_{\mathrm{OFF}}$ is predicted to be decreasing with frequency instead of being increasing with frequency, as we measured. Figure 7(b) shows that the Kondo conductance at $3.3 \mathrm{GHz}$ is higher than at 2.9 and $11.8 \mathrm{GHz}$, which is consistent with microwaves partly coupling to the gate. However, we chose not to study this mixed coupling regime, as it is not easy to quantify couplings to bias and to gate separately. Note that the thermal cycling between the measurement runs affected the coupling mode at some frequencies, possibly because wire bonds moved. In the postprocessing of the narrow peak data set, 25 frequencies were excluded starting from 86 frequencies. For the broad peak, 22 frequencies out of 59 were excluded.

\section{APPENDIX D: FROM RAW DATA TO SCALED AND NORMALIZED DATA}

The ac response of a narrow Kondo peak $\left(T_{K} \approx 30 \mu \mathrm{eV}\right)$ was studied during a first measurement run. This corresponds to data files $0-6,13-16$, and 18 and 19 in Fig. 8. From these files 5189 sets of $\left(f, V_{\mathrm{ac}, \text { source }}, G\right)$ on the Kondo resonance have been extracted and used for the analysis. After a thermal cycling the sample displayed a broad Kondo peak $\left(T_{K} \approx 95\right.$ $\mu \mathrm{eV})$. From files 9 and 12, 189 data points were extracted and used in the analysis.

Data types are explicitly indicated by the file name. They are threefold. Grayscale f2-Vac are measurements on the Kondo resonance peak, where the frequency is the sweeping loop, while the excitation voltage is the incremental loop. GrayscaleVgf2OnOff are gate sweeps for different frequencies at constant $V_{\text {ac }}$, the RF power being successively switched on and off between sweeps. Multigrayscale-VsdP2-f2 are three-loop measurements with the excitation frequency as the external loop, the excitation voltage or power as the intermediate loop, and the bias voltage as the internal loop.

We detail below how $G_{\mathrm{BG}}, G_{\mathrm{OFF}}$, and $T_{K}$ have been determined for each measurement type. The parameter values are tabulated in Fig. 8. They were used to scale the excitation amplitudes and frequencies and normalize the conductance as described in Appendix A.

\section{Grayscale f2-Vac}

The Kondo resonance at equilibrium is measured and fitted by an offset Lorentzian to obtain $G_{\mathrm{BG}}, G_{\mathrm{OFF}}$, and $T_{K}$ (see Appendix A). $V_{\mathrm{SD}}$ and $V_{g}$ are fixed on the Kondo peak during the measurement as a function of excitation frequency and amplitude.

\section{GrayscaleVgf2OnOff}

For each gate sweep, we extract the locally minimal conductance value on the Kondo ridge. This allows us to extract the Kondo conductance with and without microwave excitation for each frequency. As the Kondo conductance without excitation shifts slightly over time, we assume that $G_{\mathrm{BG}}$ varies between sweeps, while $G_{\mathrm{OFF}}$ is taken constant and is determined from fitting the Kondo resonance versus bias voltage without microwave excitation acquired before measuring GrayscaleVgf2OnOff.

\section{Multigrayscale-VsdP2-f2}

Here the equilibrium Kondo parameters are obtained for each frequency by fitting the conductance versus $V_{\mathrm{SD}}$ measured at the lowest excitation amplitude, provided that $\min \left(V_{\mathrm{ac}}\right)<$ $15 \mu \mathrm{V}$. This is justified by the observation that excitation amplitudes below this threshold do not affect the Kondo conductance compared to equilibrium. The conductance values on the Kondo peak are extracted by filtering the points with $V_{\mathrm{SD}}$ closest to the Lorentzian center fitting parameter $x_{c}$. $G_{\mathrm{ON}} / G_{\mathrm{OFF}}, h f / k_{B} T_{K}$, and $e V_{\mathrm{ac}} / k_{B} T_{K}$ are calculated with the specific fitting parameters found for the corresponding frequency. Values tabulated in Fig. 8 are averaged quantities, with standard deviation indicated in other columns.

\section{APPENDIX E: SCALED AMPLITUDE-FREQUENCY CONDUCTANCE MAPS FOR THE “BROAD” KONDO PEAK}

Figure 9 displays the experimental and ansatz scaled amplitude-frequency normalized conductance maps for the
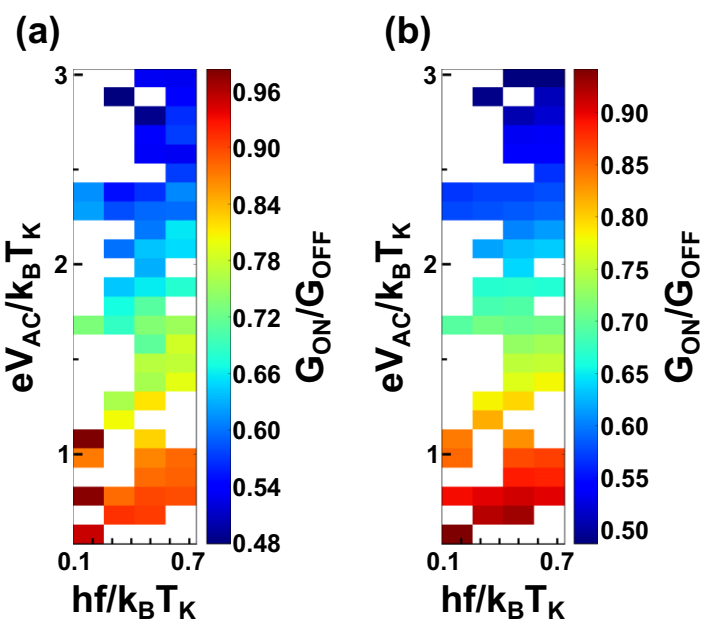

FIG. 9. (a) Experimental and (b) ansatz scaled amplitudefrequency normalized conductance map for the broad peak. 
broad Kondo peak. The higher number of blank cells compared to the "narrow" peak maps is due to the lower number of data sets in the database from which the conductance matrix is built.
[1] D. Goldhaber-Gordon, Hadas Shtrikman, D. Mahalu, D. Abusch-Magder, U. Meirav, and M. A. Kastner, Kondo effect in a single-electron transistor, Nature (London) 391, 156 (1998).

[2] J. Nygård, D. H. Cobden, and P. E. Lindelof, Kondo physics in carbon nanotubes, Nature (London) 408, 342 (2000).

[3] W. G. van der Wiel, S. De Franceschi, T. Fujisawa, J. M. Elzerman, S. Tarucha, and L. P. Kouwenhoven, The Kondo effect in the unitary limit, Science 289, 2105 (2000).

[4] Z. Iftikhar, S. Jezouin, A. Anthore, U. Gennser, F. D. Parmentier, A. Cavanna, and F. Pierre, Two-channel Kondo effect and renormalization flow with macroscopic quantum charge states, Nature (London) 526, 233 (2015).

[5] A. J. Keller, L. Peeters, C. P. Moca, I. Weymann, D. Mahalu, V. Umansky, G. Zaránd, and D. Goldhaber-Gordon, Universal Fermi liquid crossover and quantum criticality in a mesoscopic system, Nature (London) 526, 237 (2015).

[6] M. M. Desjardins, J. J. Viennot, M. C. Dartiailh, L. E. Bruhat, M. Lee, M.-S. Choi, M. R. Delbecq, A. Cottet, and T. Kontos, Observation of the frozen charge of a Kondo resonance, Nature (London) 545, 71 (2017).

[7] L. P. Kouwenhoven, S. Jauhar, J. Orenstein, P. L. McEuen, Y. Nagamune, J. Motohisa, and H. Sakaki, Observation of PhotonAssisted Tunneling Through a Quantum Dot, Phys. Rev. Lett. 73, 3443 (1994).

[8] W. G. van der Wiel, S. De Franceschi, J. M. Elzerman, T. Fujisawa, S. Tarucha, and L. P. Kouwenhoven, Electron transport through double quantum dots, Rev. Mod. Phys. 75, 1 (2003).

[9] K. Shibata, A. Umeno, K. M. Cha, and K. Hirakawa, PhotonAssisted Tunneling Through Self-Assembled InAs Quantum Dots in the Terahertz Frequency Range, Phys. Rev. Lett. 109, 077401 (2012).

[10] R. Deblock, E. Onac, L. Gurevich, and L. P. Kouwenhoven, Detection of quantum noise from an electrically driven two-level system, Science 301, 203 (2003).

[11] E. Onac, F. Balestro, B. Trauzettel, C. F. J. Lodewijk, and L. P. Kouwenhoven, Shot-Noise Detection in a Carbon Nanotube Quantum Dot, Phys. Rev. Lett. 96, 026803 (2006).

[12] T.-K. Ng, ac Response in the Nonequilibrium Anderson Impurity Model, Phys. Rev. Lett. 76, 487 (1996).

[13] J. König, H. Schoeller, and G. Schön, Zero-Bias Anomalies and Boson-Assisted Tunneling Through Quantum Dots, Phys. Rev. Lett. 76, 1715 (1996).

[14] A. Schiller and S. Hershfield, Solution of an AC Kondo Model, Phys. Rev. Lett. 77, 1821 (1996).
[15] P. Nordlander, N. S. Wingreen, Y. Meir, and D. C. Langreth, Kondo Physics in the Single Electron Transistor with ac Driving, Phys. Rev. B 61, 2146 (2000).

[16] R. López, R. Aguado, G. Platero, and C. Tejedor, Kondo Effect in ac Transport Through Quantum Dots, Phys. Rev. Lett. 81, 4688 (1998).

[17] Y. Goldin and Y. Avishai, Nonlinear Response of a Kondo System: Direct and Alternating Tunneling Currents, Phys. Rev. Lett. 81, 5394 (1998).

[18] A. Kaminski, Y. V. Nazarov, and L. I. Glazman, Suppression of the Kondo Effect in a Quantum Dot by External Irradiation, Phys. Rev. Lett. 83, 384 (1999).

[19] A. Kaminski, Y. V. Nazarov, and L. I. Glazman, Universality of the Kondo effect in a quantum dot out of equilibrium, Phys. Rev. B 62, 8154 (2000).

[20] B. Béri and N. R. Cooper, Topological Kondo Effect with Majorana Fermions, Phys. Rev. Lett. 109, 156803 (2012).

[21] J. M. Elzerman, S. De Franceschi, D. Goldhaber-Gordon, W. G. van der Wiel, and L. P. Kouwenhoven, Suppression of the Kondo effect in a quantum dot by microwave radiation, J. Low Temp. Phys. 118, 375 (2000).

[22] A. Kogan, S. Amasha, and M. A. Kastner, Photon-induced Kondo satellites in a single-electron transistor, Science 304, 1293 (2004).

[23] K. Yoshida, K. Shibata, and K. Hirakawa, Terahertz Field Enhancement and Photon-Assisted Tunneling in Single-Molecule Transistors, Phys. Rev. Lett. 115, 138302 (2015).

[24] B. Hemingway, S. Herbert, M. Melloch, and A. Kogan, Dynamic response of a spin-1/2 Kondo singlet, Phys. Rev. B 90, 125151 (2014).

[25] P. W. Anderson, A poor man's derivation of scaling laws for the Kondo problem, J. Phys. C 3, 2436 (1970).

[26] P. K. Tien and J. P. Gordon, Multiphoton process observed in the interaction of microwave fields with the tunneling between superconductor films, Phys. Rev. 129, 647 (1963).

[27] J. J. Viennot, J. Palomo, and T. Kontos, Stamping single wall nanotubes for circuit quantum electrodynamics, Appl. Phys. Lett. 104, 113108 (2014).

[28] L. I. Glazman and M. Pustilnik, Coulomb blockade and Kondo effect in quantum dots, arXiv:cond-mat/0302159.

[29] P. Jarillo-Herrero, J. Kong, H. S. J. Van Der Zant, C. Dekker, L. P. Kouwenhoven, and S. De Franceschi, Orbital Kondo effect in carbon nanotubes, Nature (London) 434, 484 (2005). 